\title{
Volumetric Bone Maintenance of Guided Bone Regeneration in Implant Dentistry
}

IMPLANT THERAPY OUTCOMES, SURGICAL ASPECTS
Teixeira Araújo, P.; Ribeiro Sampaio, N.; Oliveira, R.; Escobar, T.; Alfaiate, D.; Matos Dias, F. SPI Research Centre

\section{Abstract}

Background: Several different techniques to achieve bone volume have been used with success over the years. These techniques have allowed the
rehabilitation of patients with dental implants that otherwise wouldn't be rehabilitation of patients with dental implants that otherwise wouldn't be areas subject to Guided Bone Regeneration from $61.6 \%$ to $100 \%$.

Aim: This poster aims to present a scientific review about the volumetric bone maintenance through time using different techniques.

Materials/Methods: Inclusion and exclusion criteria were defined before the beginning of the review. Criteria included studies published in the English language, published between 2005 and 2015, number of participants, type and area of intervention, follow-up period and outcome parameters. The search strategy involved searching the Pubmed electronic data base with different combinations of key words: "Dental", "Implants" and "GBR". A review was performed to study the information and conclusions on the studies
included and observe the behaviour of the bone through time at regenerated sites.

Results: The search resulted in four studies that were included, one systematic review, two randomized controlled clinical trial and one prospective study totalizing 1534 patients treated with 3166 implants using different techniques and approaches to increase alveolar bone dimensions vertically and horizontally. The results failed to demonstrate the superiority of any one technique over another during the period of time of observation. One
should notice however the large amount of vertical bone gain achieved when shing distraction osteogenesis technique. According to the results a using distraction osteogenesis technique. According to the results a
maximum of $7.08 \mathrm{~mm}$ of bone gain can be achieved for vertical augmentation with distraction osteogenesis whilst a maximum of $4.3 \mathrm{~mm}$ of bone gain can be achieved for horizontal augmentation using bone blocks/grafts.

Conclusions: Within the limitations of this review it appears that all techniques used for augmentation procedures can be successful for the period of observation (a maximum of two-stage approach. In regards to the materials used for these procedures

\section{Background and Aim}

It has been 45 years since dental implants were first used to replace a missing teeth and first reported (1). Since then the use of dental implants to rehabilitate partially dentate or edentulous patients has become a routine treatment option for many clinicians which is well documented in the literature $(2,3,4,5,6)$. It is also considered to be a predictable treatment with predictable reported that in general, implants placed in healed sites had an overall survival rate at 5 years of $95 \%$ and that at 10 years this percentage was higher than $90 \%$. A prerequisite to successfully rehabilitate patients with dental implants is an adequate interface between the implant and the surrounding bone, and successful osseointegration (10). A lack of sufficient bone tissues will adversely affect the long-term prognosis of the implant and subsequent rehabilitation $(3,11)$ and this may manifest itself in either the short or long term. The implantologist frequently faces situations where this is the case, and can either be due to trauma, tooth loss, chronic intection or periodontal disease $(12,13)$.

The development of guided bone regeneration (GBR) techniques first appeared in the late 1980 's with a series of experimental studies. This non-resorbable barrier membranes to create and stabilize a space into which cells originating from host bone tissues can grow into any blood clot without the interference of the faster proliferating soft tissue cells, and convert into bone tissue. In some situations this allows the insertion of dental implants into areas of alveolar deficiency which otherwise would not be possible (16). Several different techniques have been developed over the years that can help reconstruct deficient alveolar indges and that permit the implant placement either in a simultaneous or staged approach (12).

Among the techniques that allow the clinician to increase alveolar bone dimensions vertically and horizontally are: GBR; bone block grafts; distraction jaws; and techniques several different materials can also be used.

This poster aims to present a scientific review about the volumetric bone maintenance through time using different techniques.

\section{Methods and Materials}

Inclusion (English publications in dental literature; studies in healthy adults; minimum of 10 participants; studies with a mean follow-up in time over 12 implants) and exclusion (studies with less than 10 participants; sinus lift augmentation studies: studies not fulfilling the inclusion criteria; studies providing only histological data; studies describing bone augmentation techniques as a treatment option following peri-implantitis, trauma, tumor ablation or therapy for various medical syndromes) criteria were defined before the beginning of the review by the authors. The search strategy involved searching the Pubmed electronic database using different combinations of the following keywords: "Dental", "Implants" and "GBR". Criteria were limited to studies published between 2005 and 2015 . The
search was limited to systematic reviews, randomized and non-randomized search was limited to systematic reviews, randomized and non-randomized evaluated in three cycles in order to assess their suitability for this review. Firstly, all the titles were screened to eliminate irrelevant publications, animal studies and review articles. Secondly, all abstracts of publications selected during the first screening were analysed, excluding studies based on the exclusion criteria - number of patients, the type of intervention and follow-up
after implant placement. Finally, full texts were assessed to further evaluate their suitability for inclusion

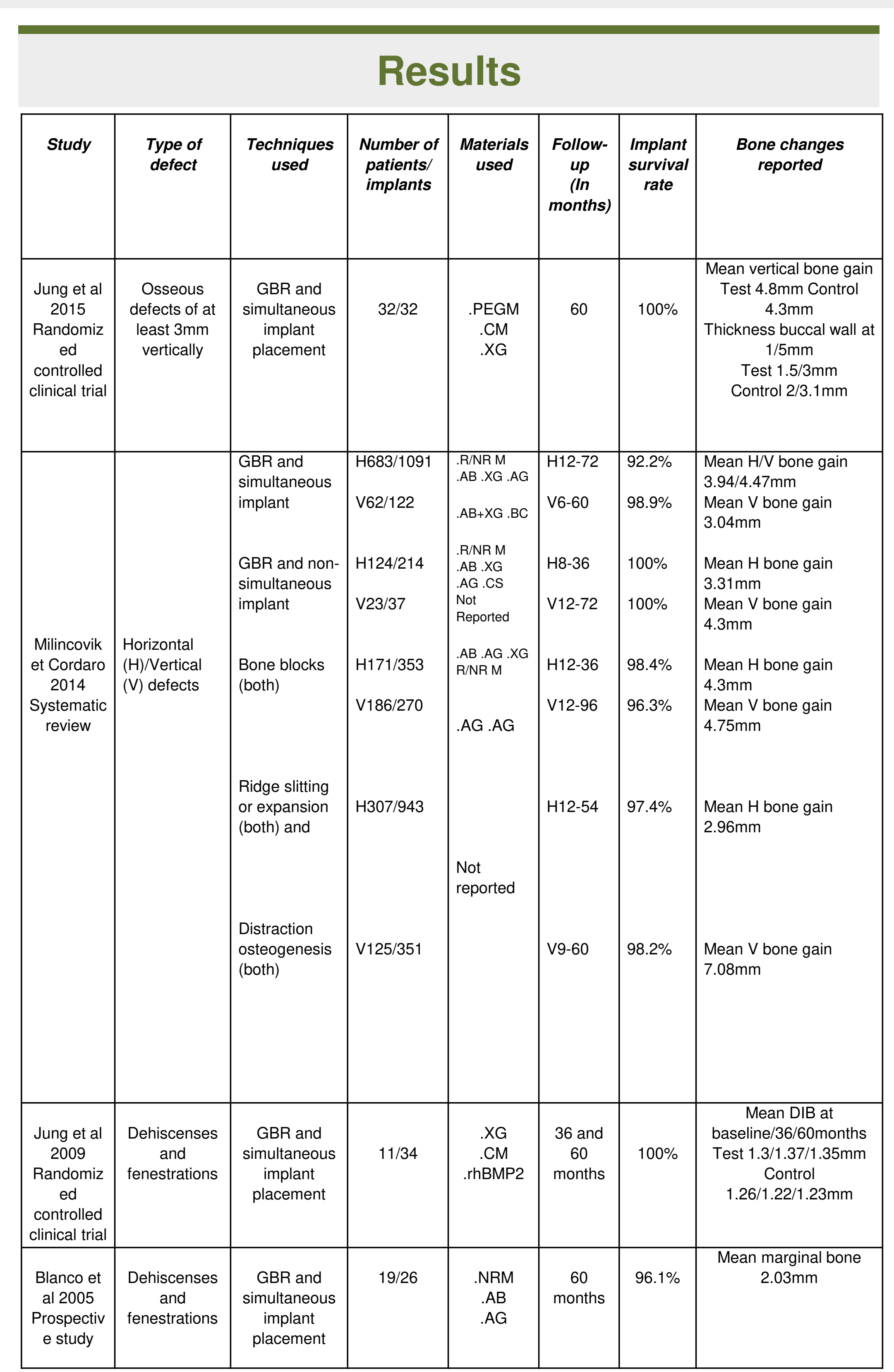

Table1. Information collected from the studies. Legend: Autograft, Ab; Xenograft, XG
Allograft, AG; Blood clot, BC; Collagen sponge, CS; R A sorbable membrane. RM; Non
resorbable membrane, NRM; Collagen membrane, CM; PEG membrane, PEGM.

\section{Conclusions}

This review revealed that different augmentation techniques with different It is difficult to make a direct comparison between the four studies as only two were the results presented as vertical bone gain and horizontal bone gain
(Jung et al 2015 , Milinkovic et Cordaro 2014 ). Although the other studies refer to the results the same way, as "mean marginal bone level" these were
calculated differently which makes a comparison difficult. In light of the results gathered it appears that there is no clear indication of a better technique over another. All the techniques studied present challenges regenerate bone in partially edentulous patients and allow implant placement some materials can help in reducing the treatment time frame: Overall, GBR and successful outcomes regarding bone gain and maintenance and with
high implant survival rate in time. More studies should be conducted with normalized rules describing the
clinical situation before and after the augmentation procedure to allow a
better interpretation and comparison of the results in the different studies.

\section{References}

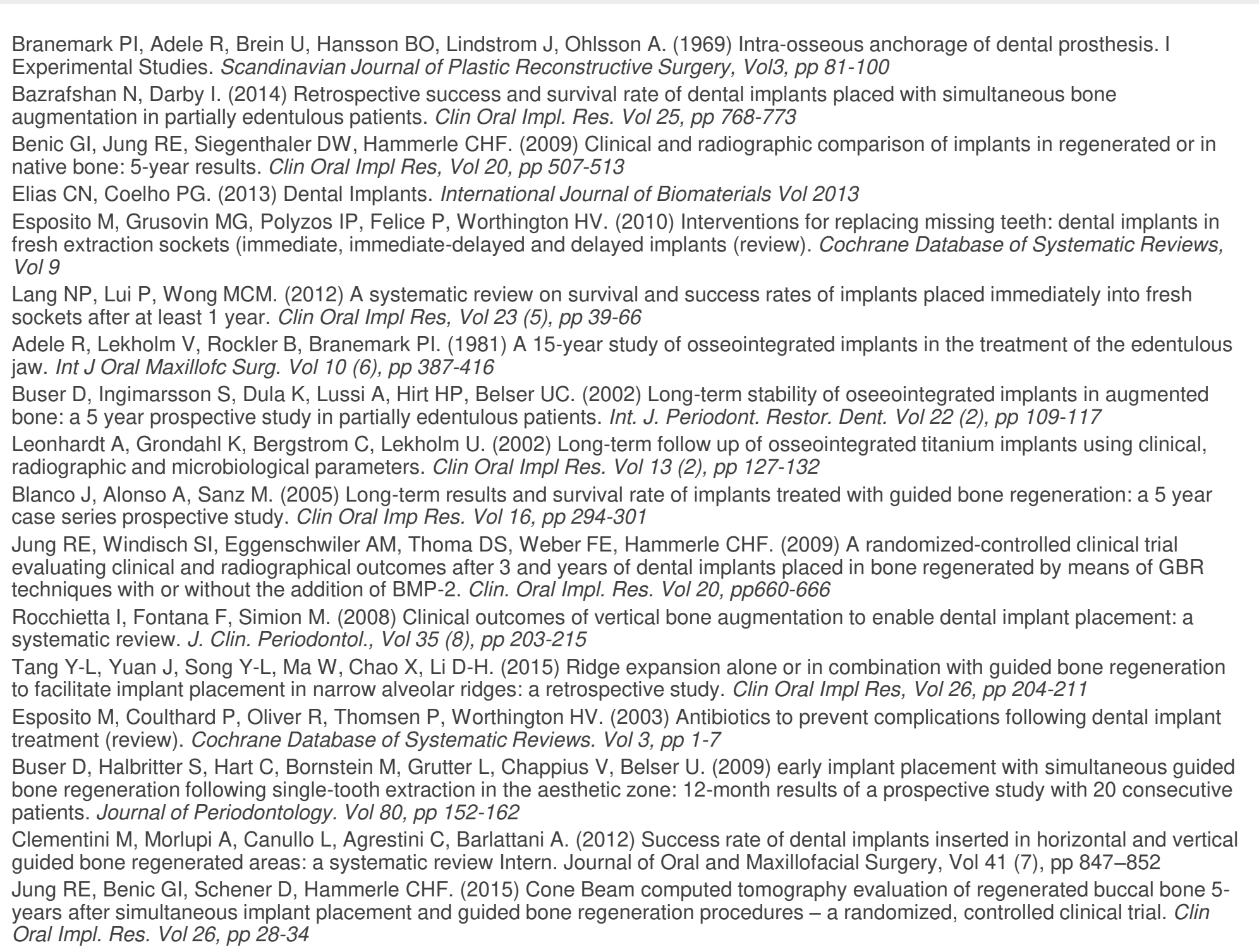

